Original Research Paper

\title{
Research of the Process of Shaping Folded Filler of Sandwich Panels from Cardboard
}

\author{
Il'dus Zakirov, Alexandr Nikitin, Alexandr Naumov and Nargiz Faizullina \\ Department of Strength of Structures, \\ Kazan National Research Technical University Named After A.N. Tupolev, Kazan, Russia
}

\author{
Article history \\ Received: 07-10-2015 \\ Revised: 23-11-2015 \\ Accepted: 24-11-2015 \\ Corresponding Author: \\ Il'dus Zakirov \\ Department of Strength of \\ Structures, Kazan National \\ Research Technical University \\ named after A.N. Tupolev, \\ Kazan, Russia \\ Email: nurzak@mail.ru
}

\begin{abstract}
Sandwich panels with the corrugated filler made of composite cardboard are used in building constructions, road sound dampening screens, etc. Taking into account that in industries applying sandwich panels, the demand for such filler is relatively high, the competitiveness of the product must be at a high level. This requires developing a highperformance industrial technology to produce it. These production processes include rotary shaping. The well-known technology for manufacturing corrugated cardboard from thin paper does not allow making very high products. The article considers the possibility of producing relatively high corrugated filler by creasing and folding. The important point here is to make a flat pad, as one of the elements of the corrugation, at the point of its contact with the skins of the panel. We studied two schemes of folding a preliminarily creased cardboard web and evaluated the advantages of these schemes. We suggested the creaser shape and structure, which allow making high-quality connect pads on the corrugated cardboard within a wide range of sizes.
\end{abstract}

Keywords: Composite Cardboard, Corrugation, Creasing, Folding, Flat Pad

\section{Introduction}

Sandwich panels with folded cardboard filler are used in elements of building structures, interior walls, doorframes, sound dampening screens, etc. In terms of consumer qualities, folded filler made by industrial technology is efficient. One of such technologies is the rotary shaping process, which includes the creasing and folding operations.

In the case under study, creasing is the prior to folding operation of the material deformation in the area of creasing along the crease edges set by drawing. Introduction of this operation ensures good quality of the product when folding and considerably reduces the amount of the required bending momentum.

The above folding operations are a kind of bending operation. After creasing, the folding can be done in two ways: The first one is bending towards the direction of the deepening made by creasing-the direct folding pattern, the second one is bending in the opposite direction-it is done with the reversion of the material-the reverse pattern.

Numerous papers are devoted to the study of the cardboard creasing and folding operations. However, these studies are mainly focused on solving issues related to the production of packaging products and office supplies. Such works include the studies of S. Nagasawa and his colleagues (2003), who obtained the characteristics of creasing and folding when changing the depth of creasing and the lateral deviation of the creaser. In their work, they also presented the results of the effect of the nominal shear deformation (creasing depth) on the change of residual stiffness and the factor of hysteresis of the bending momentum.

Mentrasti et al. (2013) studied theoretically and experimentally the value of the bending moment at folding after creasing depending on the angle of bending and gave recommendations on the parameter selection. Based on experimental and computer research, Beex and Peerling (2009) identified the regularities in the process of cardboard lamination during creasing and folding.

It is known that polymeric paper and cardboard have considerable anisotropy. Barbier et al. (2006) using the finite-element method showed that the highly elastic anisotropy leads to a decrease in the level of deformation during folding. This effect had not been taken into account in previous works. Mäkelä and Ostlund (2003) developed an orthotropic visco-elastic model to simulate the behavior of paper at shaping. They presented numerical implementation of the model and showed that 
the model is in good agreement with the experimentally observed mechanical behavior of paper.

However, as has been noted, a study of creasing and folding was carried out with respect to the manufacture of products, in which the folding operation was performed by bending and the manufacture of which did not require making a flat pad after creasing and folding on the horizontal ridges of the corrugation.

At industrial shaping of the folded cardboard filler, the creasing and folding operations are performed using a rotating machine that has some peculiar features in the formation of creasing and selection of the folding pattern. Firstly, folding is made by profiling and, secondly, in contrast to the known studied patterns, folding is performed according to the direct pattern. The well-known works consider the operation of reverse folding.

This work studies the influence of the following parameters on the width of the resulting flat pad when folding a creased sample: The cardboard thickness, the size of the creaser, the depth of penetration of the punch with the sample into the die cavity. There were two options of folding.

The aim of the article is to determine the advantages of making a flat pad in the crease area when folding according to the direct and reverse patterns.

\section{Proposed Methodology}

\section{An Analysis of the Creasing and Folding Patterns}

The analysis shows that the shape and size of creasing have a significant impact on the desired result when folding. In turn, the selection of the folding pattern also influences the dimensions of the corrugation in the creasing area, as well as the product quality. Table 1 shows the flow charts of creasing and folding that we analyzed.

The first row of Table 1 shows the patterns of direct and reverse folding of the semi-finished corrugated web made by creasing by means of imbedding the tool in the surface of the work piece held on a flat hard base. In the middle of the row, the creasing pattern with the tool having a cylindrical working portion of radius $\mathrm{R}$ is shown. Creasing is performed to depth $h$. On the left and right, the shapes of products after folding according to the direct and reverse patterns respectively are shown.

By the stress-strain state, the pattern of reverse folding is preferable, as the layers that are stretched due to the increased parameter of the stretched portions suffer less stretch tension at creasing and are less susceptible to damage when folded. The external surface area of deformation in both patterns has a pronounced curvilinear contour.

In the second row of the table, the pattern of creasing with a tool that has width $\mathrm{P}$ is shown. The working edge of this tool has a straight portion in the cross-section with ends rounded to the radius $\mathrm{R}$. Creasing is carried out in the die that has the width $B=n+2 s$, to the depth $\mathrm{H}$ that is no more than two thicknesses of the work piece.

If the folding is performed according to the direct pattern, here on the outer surface of the crease area a flat pad is created with width being:

$b=P+2 s-2(R+s)$

In the case of reverse folding, the pad is wider due to the rectification of the corner area:

$$
b=P+\pi(R+s / 2)
$$

In the middle of the last line of the table, the creasing option is shown when due to the greater movement of the creaser, a wall with height (H-s) is created. For this creasing option, direct folding leads to the formation of the flat portion, the width of which is defined by the above dependence (1).

The width $b$ of the flat portion in the case of reverse folding increases due to the presence of the wall of a certain height:

$$
\begin{aligned}
& b=P-2 R+(\mathrm{R}+s / 2) \pi+2\left(\mathrm{~h}-\mathrm{R}-\left(\mathrm{R}_{M}+s / 2\right) \pi / 2\right. \\
& =P+(R+s / 2) \pi-4 R-\left(\mathrm{R}_{M}+\frac{s}{2}\right) \pi / 2+2 h
\end{aligned}
$$

It should be noted that at direct folding (second and third rows) in the corner areas of the inner side of the crease, there is compression of the cardboard layers and due to shear stresses-lamination, instability and buckling.

When we fold according to the reverse patterns shown in the second and third rows of the table (the third column), the compressive stresses are concentrated on the back side of the pad formed outside. The outer layers are subjected to significant stretching, as the inner layers are laminated, which results in buckling and consequent bulging.

The patterns provided in Table 1 and their analysis are based on our experience increasing research and require experimental validation.

Thus, it is necessary to conduct experiments to obtain data for the assessment in formulas (1) and (2) of the complete accounting of the factors influencing the formation of the pad width, as well as to evaluate visually the quality of the shape of the deformation area made by creasing and folding and to develop proposals for the most efficient patterns of creasing and folding.

\section{Experimental Study}

\section{Experimental Device}

Figure 1 shows the design of a device for creasing of experimental samples. 


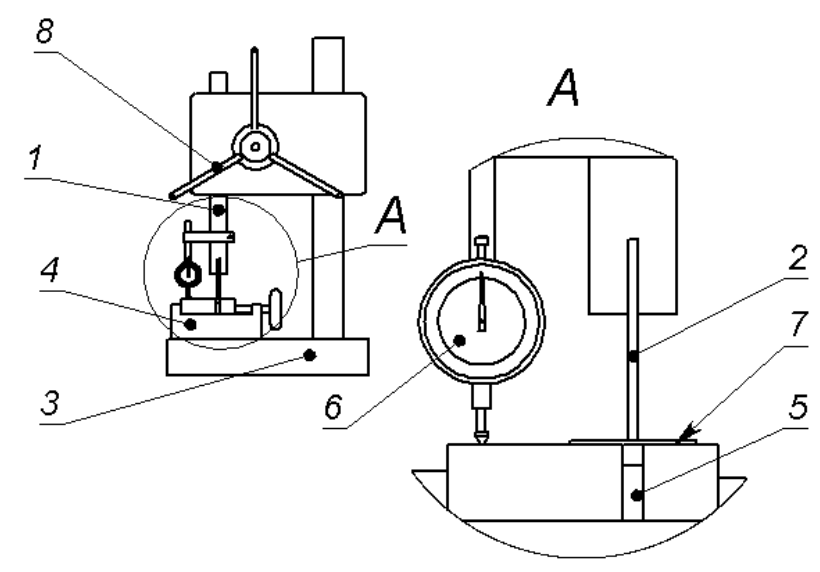

Fig. 1. The experimental creasing device

The device has movable rod 1 with a socket for mounting punch 2 of the creaser. On the bottom plate of rack 3 , there is an element of the creaser-sectional die 4 . Replaceable bushes 5 serve to adjust the alignment of the die to the specified size. Dial indicator 6 fixed on movable rod 1 serves to control the working stroke of punch 2 .

As the creaser, we used a set of punches made of thick steel plates that had thickness $\mathrm{P}$ equal to, respectively, 1, 1.5, 2 and $2.5 \mathrm{~mm}$. For the experiments, we used cardboard of K-1 grade $0.5 \mathrm{~mm}$ thick. The samples' dimensions were $17 \times 50 \mathrm{~mm}$. The samples were folded manually.

Measuring of the sample's dimensions after creasing and folding was performed on a device, the photo of which is shown in Fig. 2.

The device comprises unit 1 , which serves to hold the sample based on a side edge and includes digital microscope 2, used to photograph the sample and display the information on monitor 3 of the computer, where the required parameters of its shape were measured.

\section{Experimental Observations}

The first problem, which was solved by performing the experiments, is the analysis of the patterns shown in Table 1, using the experimental results, as well as verification of the calculated dependence (2). The second task was based on the solution of the first task and was to analyze and give a proposal for the most efficient pattern of the technology for corrugated products with the given values of the connection pad and shaping corrugated web with relatively high corrugations for cardboard fillers.

The experiments were carried out on the device (Fig. 1) as follows.

Punch 2 of the creaser with width $\mathrm{P}$ was fixed on movable rod 1 . Sectional die 4 was set to the width $\mathrm{V}=$ $\mathrm{P}+2 \mathrm{~s}$ by installation of removable liner 5 . The depth of the die cavity in this case was $2 \mathrm{~mm}$. Indicator 5 was set to zero in the position of contact of the working edge of punch 2 with the plane of the sample.

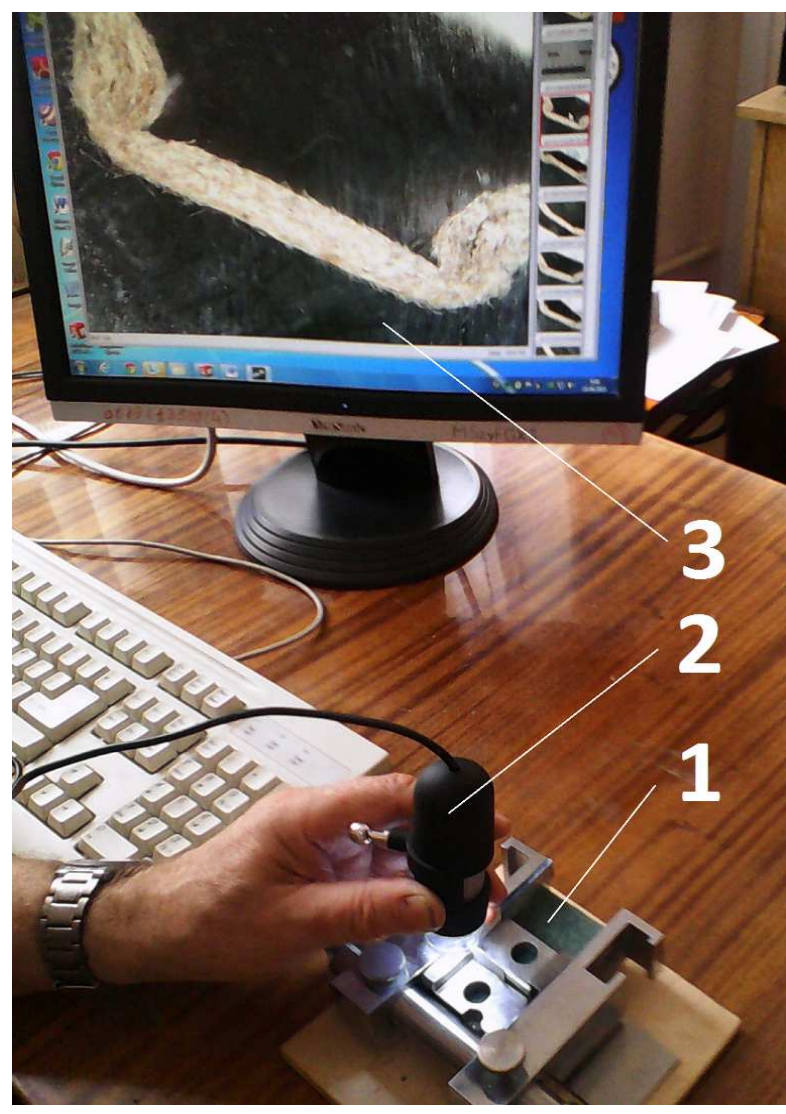

Fig. 2. The device for measuring the pad width after folding

For this, we used a steel probe, the thickness of which was equal to the thickness of the sample material, after which the punch was raised. Sample blank 6-a strip of cardboard $0.5 \mathrm{~mm}$ thick that had size in plane $17 \times 50 \mathrm{~mm}$-was placed on the die surface, the creaser by rotating winch 7 was moved to its surface and then lowered to the predetermined value $\mathrm{N}$ with the movement controlled by indicator 6 to the accuracy of $0.01 \mathrm{~mm}$. Thereafter, the punch was retracted to the starting position and the sample was removed from the die.

Three samples were creased sequentially with the same settings and depth. The samples with the same values of the punch and die widths were subjected to creasing with the penetration depth of 1.0,2.0 and $2.3 \mathrm{~mm}$.

Two of the three samples were subjected to folding at angle $180^{\circ}$-the first one according to the direct pattern, the second one according to the reverse pattern and the third remained in the position after creasing. The folded samples after removal of the load rebounded to angle $90^{\circ}$ under the effect of the internal momentum.

Each sample was placed in the measuring fixture (Fig. 2) with the angle between the faces fixed at $90^{\circ}$, where we photographed with a digital microscope the crease area and measured the length of the flat portion.

Figure 3 shows images of samples' sides, which were creased and folded according to the patterns shown in Table 1. 

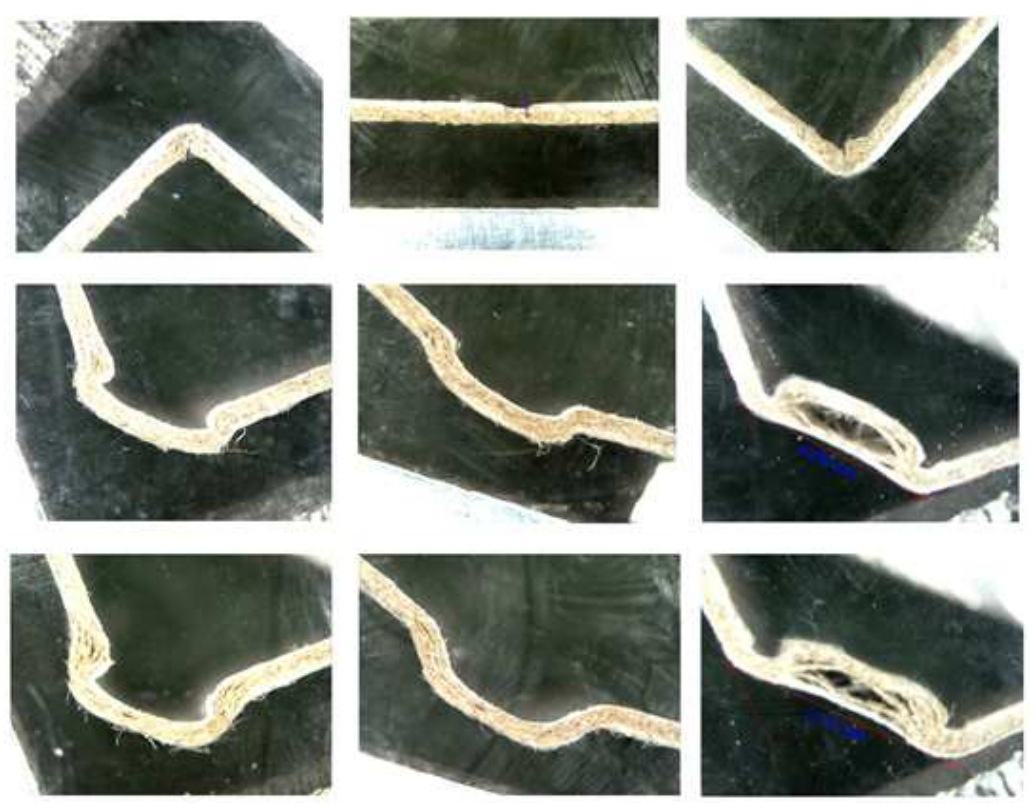

Fig. 3. Photos of samples after creasing and measurement of the width of the flat portion after folding in accordance with the patterns provided in Table 1

Table 1. Creasing options and the shape of samples after folding

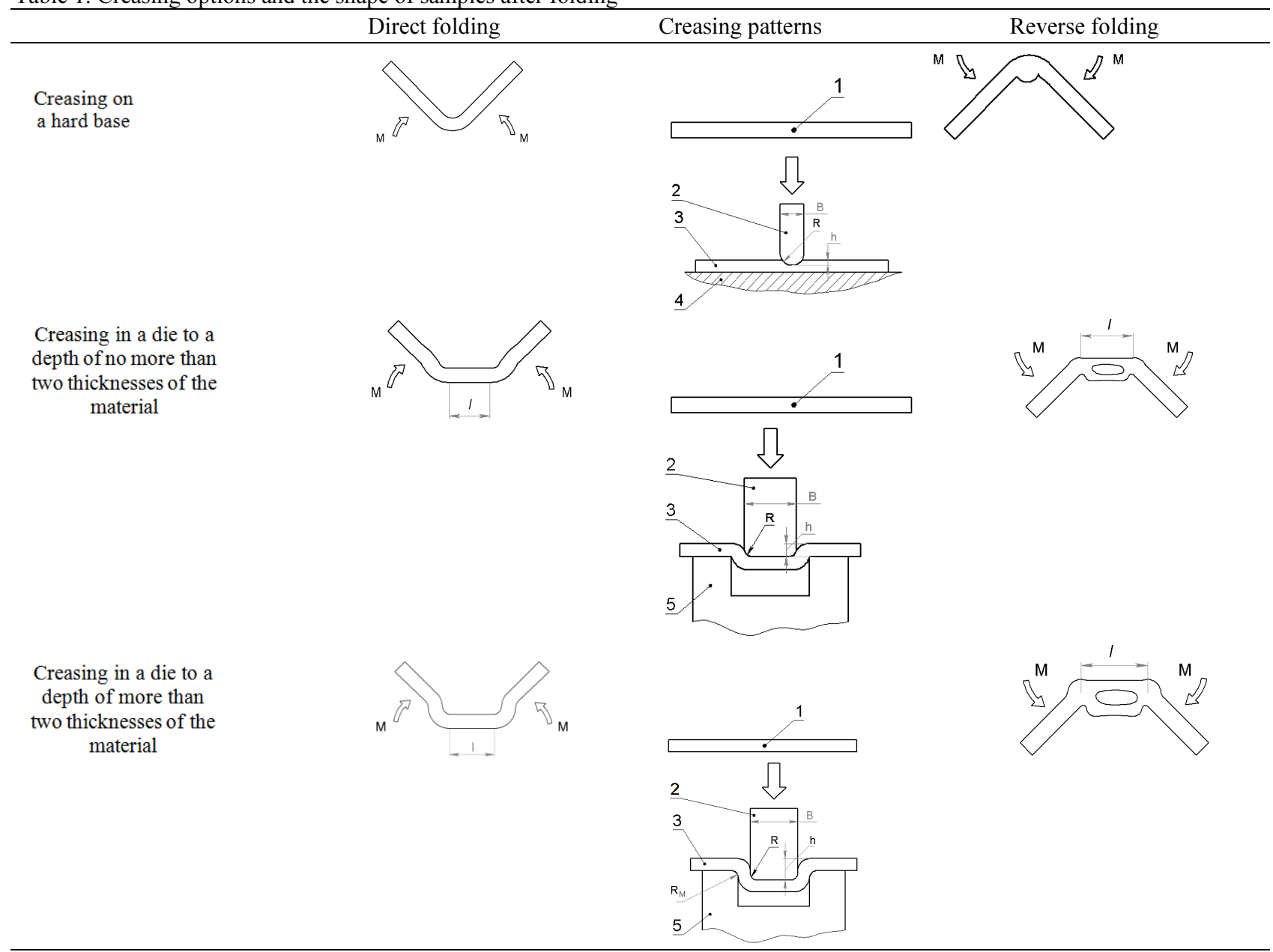


The sample shown in the first image in the first row was subjected to creasing with the tool $1.0 \mathrm{~mm}$ in width and $0.5 \mathrm{~mm}$ in radius of the curvature of the working edge. The creasing depth was $h=0.12 \mathrm{~mm}(\sim 25 \%$ of the thickness). In the middle of the row one can see the sample after creasing. The right photo demonstrates the sample after direct folding. It can be seen that the contour of the outer surface in the bending area is convex and circular and on the inner side due to compression of the layers, a bulge appeared. The left photo of this row shows the sample after reverse folding. Here on the outer side, the contour in the crease area has a more regular shape, as the conditions of shaping of the outer layers are more favorable because there is a reserve contour in the creasing area. Definitely, we can note that in both patterns, no visible pad is formed.

In the second row, there are the images of the samples, which were subjected to creasing with the tool $2.5 \mathrm{~mm}$ in width in the die $2.0 \mathrm{~mm}$ in depth to the depth of $1.0 \mathrm{~mm}$. The picture in the middle shows the sample after creasing and removal from the die. The left picture shows the sample after direct folding to the parallel position of the faces and removal of the deforming load. As a result of rebounding, the angle between the faces reduced to about $90^{\circ}$.

Folding was made on the corners of the crease. On the inner surface in the area of occurrence of compressive stresses, bumps (bulges) with noticeable material lamination appeared. On the outer surface, a pad of width $b=3.15 \mathrm{~mm}$ appeared with a noticeable bulge. On the right, there is a picture of the sample after reverse folding. On the outer surface, there is a flat plane 3.96 $\mathrm{mm}$ in width. The bending at folding occurs on the corners. At noticeable stretching of the outer layers and compression of the inner ones, the shear stresses result in lamination of the material and buckling.

The samples in the third row were creased with the same tool as the second series' samples, but the

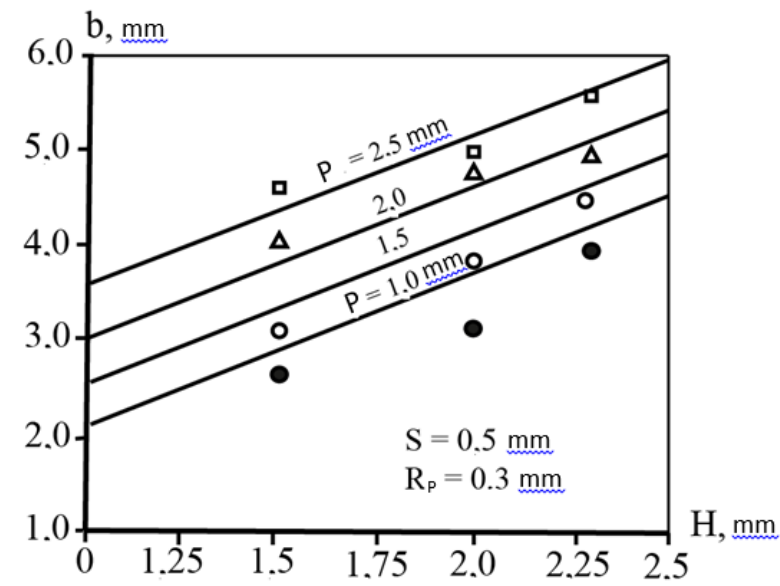

(a) penetration depth was increased to $\mathrm{h}=2.25 \mathrm{~mm}$. Therefore, in this case, part of the creasing surface is shaped as a vertical "wall". On the middle picture, the creased sample is shown after the load removal, so the "wall" after rebounding is not perpendicular to the bottom part of the crease. On the left, the sample after direct folding is shown. The outer side of the crease portion appears as a protrusion with the height equal to $h$ and a pad with a relatively proper regular plane of width $\mathrm{b}=3.13 \mathrm{~mm}$. The picture on the right shows the sample folded according to the reverse pattern. Bending at folding takes place at the corners; however, at dragging out, the bottom part of the crease is stretched. The material of the inner surface under the action of the compressive and shear deformations is laminated and bulged. On the outer surface of the crease area, a pad is formed. In this case, its width is $b=4.82 \mathrm{~mm}$. The surface of the pad has a deflection, the value of which depends on the recorded value of the folding angle.

Figure $4 \mathrm{a}$ shows charts of dependence of the width $\mathrm{b}$ of the flat portion on the penetration depth $\mathrm{H}$ calculated by the formula (2) with the width of the creaser equal to 1 , $1.5,2$ and $2.5 \mathrm{~mm}$ and the radius $\mathrm{R}$ of the chamfers equal to $0.3 \mathrm{~mm}$. It also shows the experimental values of the parameter $b$. The range of deviations of the experimental values from the calculated ones is within $0-15 \%$.

The information presented in these graphs concerns samples subjected to reverse folding. Figure $4 \mathrm{~b}$ shows the charts calculated with respect to the creaser with nonchamfered edges of the working part.

As can be seen from the comparison of the charts in Fig. 4a and 4b, the radius of the tool's edge chamfering significantly affects the dimension $b$ of the flat area after folding.

At direct folding, as the structure of the formula (2) shows, as well as the results of experiments, the penetration depth $\mathrm{H}$ does not affect the dimension $\mathrm{b}$ of the flat pad and has the shape of a bulge (Fig. 3).

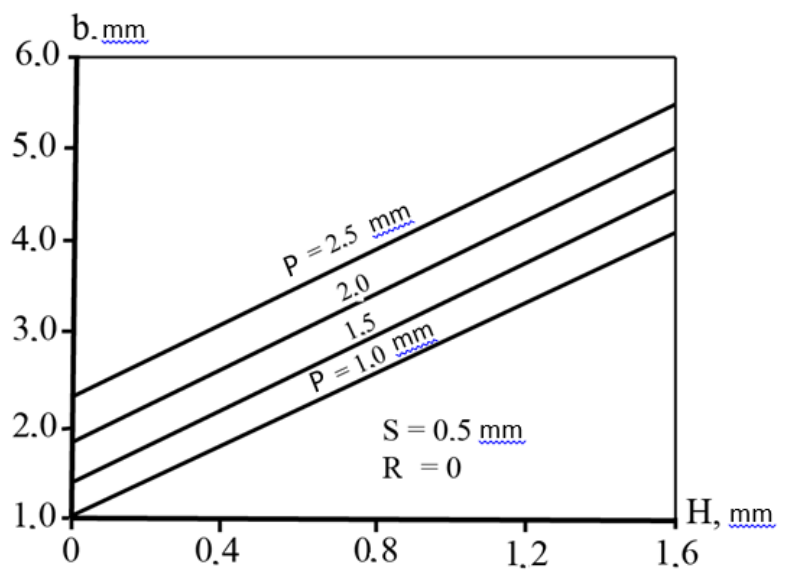

(b)

Fig. 4. Charts of the dependence of width $\mathrm{b}$ of the flat portion on the penetration depth $\mathrm{N}$ 


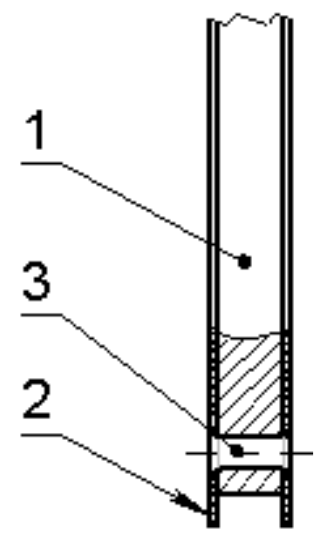

Fig. 5. The option of the design of the creaser for broader creasing: 1-core; 2-creasing knife; 3-rivet

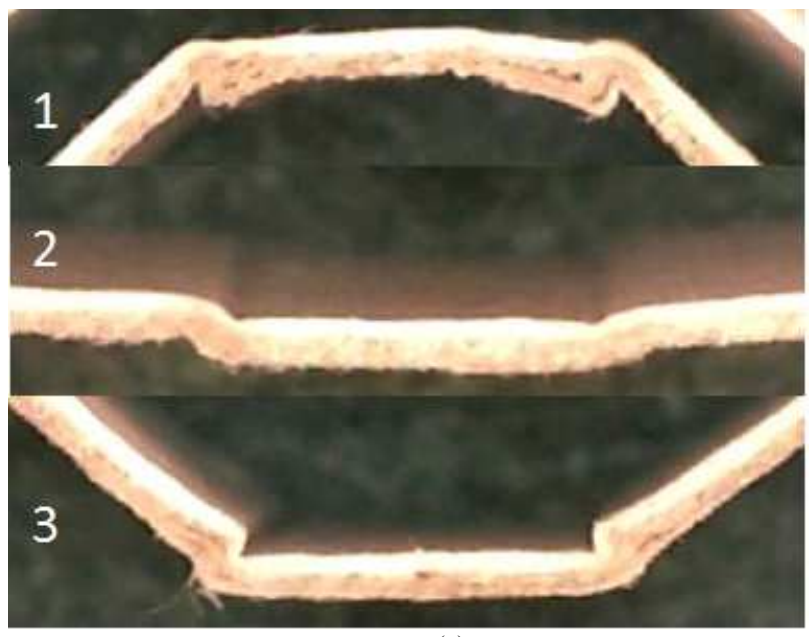

(a)

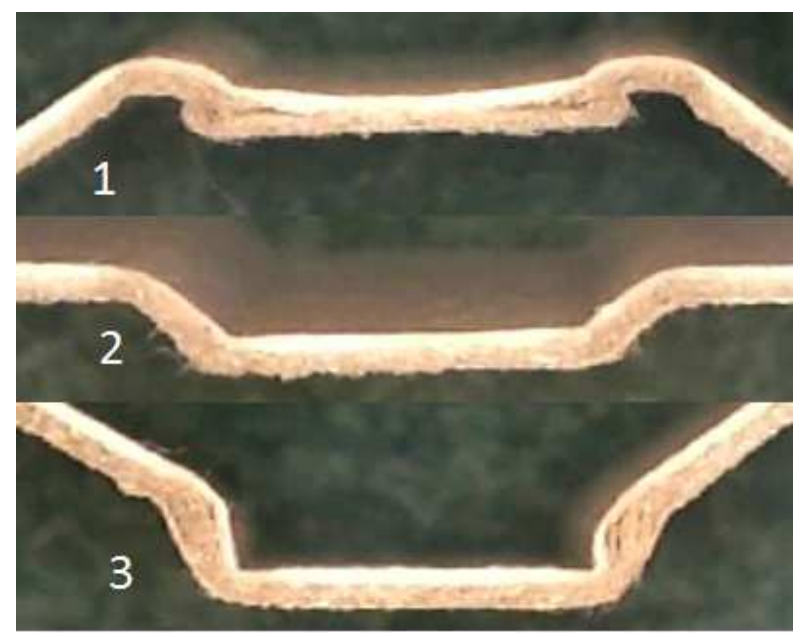

(b)

Fig. 6. Pictures of the samples with creasing to the depth h: a-1.0 mm, b-2.25 mm 1-direct folding; 2-the sample after creasing; 3reverse folding

Thus, the analysis of the patterns conducted based on the performed experiments leads to the conclusion that from the point of view of the formation of flat surfaces, the direct pattern of folding with the creasing option that involves penetration of the creaser to the depth $\mathrm{H}$ that does not exceed two thicknesses of the product's material and the reverse pattern with the options involving the penetration depth $\mathrm{N} \leq 2 \mathrm{~s}$ and $\mathrm{N} \geq 2 \mathrm{~s}$ are promising. However, it should be noted that the requirements to increase the width of the flat portion along the corrugation edge, for example, to enhance the strength of connection of the corrugated web with the panel's skin, lead to the need to increase the width of the punch and, respectively, to increasing the required force of creasing. To reduce the load on the creaser, a composite structure of the punch was proposed, the shape of which is shown in Fig. 5.

The deficiency of the reverse pattern is that there are certain difficulties in its implementation. Given the fact that the direct pattern of folding is technologically simpler at the implementation of shaping the corrugated filler, in particular, additional experiments were performed using rotary equipment to determine the feasibility of the pattern.

To this end, four composite creasing punches were produced with the wider width $\mathrm{P}$, respectively: $2.7,3.2$, 4.2 and $5.2 \mathrm{~mm}$, the design of which is shown in Fig. 5. At the same time, the side plates $0.6 \mathrm{~mm}$ in thickness constituting the working part of the tool were made without chamfers. The mate (die) was adjusted to the width $\mathrm{P}+2 \mathrm{~s}$ and depth of 0.5 and $2.0 \mathrm{~mm}$.

The performed experiment that involved using this rig confirmed our assumption that the creasing punches in the width range between 2.7 and $5.2 \mathrm{~mm}$ with the creasing knives $0.6 \mathrm{~mm}$ thick at the depth of penetration $\mathrm{H}=1 \mathrm{~mm}$ and $\mathrm{H}=2.25 \mathrm{~mm}$ provide formation of crease with a flat bottom across the width of the creasing punch. These samples after direct folding have flat 
portions without any noticeable defects and disruptions of flatness in the crease area on the outer side. In the case of reverse folding, this surface at the depth of penetration of $1 \mathrm{~mm}$ has a deflection and at the depth of penetration of $2.25 \mathrm{~m}$-clearly expressed two humps.

Figure 6 shows photographs of samples with the crease performed with the penetration of the creaser $5.2 \mathrm{~mm}$ in width, respectively, to the depth of 1 and $2.25 \mathrm{~mm}$.

The folding was carried out according to the direct and reverse patterns. The photos show that the samples after direct folding have a good marketable appearance on the flat portions.

\section{Conclusion}

The performed study allows making the following conclusions:

- The direct and reverse folding patterns using the creasing operation enable making flat portions in the area of the crease, which allows intensifying the process of corrugated products shaping

- The most promising is the creasing pattern, which uses a composite scoring punch followed by direct folding

\section{Acknowledgement}

The author thanks Kazan National Research Technical University for supporting this research.

\section{Funding Information}

The author thanks Kazan National Research Technical University for supporting this research.

\section{Author's Contributions}

Il'dus Zakirov: Designed the research plan and organized the study.

Alexandr Nikitin: Participated in all experiments, coordinated the data-analysis.

Alexandr Naumov: Coordinated the data-analysis and contributed to the writing of the manuscript.

Nargiz Faizullina: Contributed to the writing of the manuscript and coordinated the mouse work.

\section{Ethics}

The authors have no conflicts of interest in the development and publication of current research.

\section{References}

Barbier, C., P.L. Larsson and S. Östlund, 2006. On the effect of high anisotropy at folding of coated papers. Composite Structures, 72: 330-338.

DOI: 10.1016/j.compstruct.2005.01.003
Beex, L.A.A. and R.N.J. Peerlings, 2009. An experimental and computational study of laminated paperboard creasing and folding. Int. J. Solids Structures, 46: 4192-4207. DOI: $10.1016 /$ j.ijsolstr.2009.08.012

Mäkelä, P. and S. Östlund, 2003. Orthotropic elasticplastic material model for paper materials. Int. J. Solids Structures, 40: 5599-5620. DOI: 10.1016/S0020-7683(03)00318-4

Mentrasti, L., F.Cannella, M. Pupilli and J.S. Dai, 2013. Large bending behavior of creased paperboard. I. Experimental investigations. Int. J. Solids Structures, 50: 3089-3096. DOI: 10.1016/j.ijsolstr.2013.05.018

Nagasawa, S., Y. Fukuzawa, T. Yamaguchi, S. Tsukatani and I. Katayama, 2003. Effect of crease depth and crease deviation on folding deformation characteristics of coated paperboard. J. Mater. Proc. Technol., 140: 157-162. DOI: $10.1016 / \mathrm{S} 0924-0136(03) 00825-2$ 